\title{
Cost-effectiveness of a text message programme for the prevention of recurrent cardiovascular events
}

\author{
Edward Burn, ${ }^{1}$ Son Nghiem, ${ }^{1}$ Stephen Jan, ${ }^{2}$ Julie Redfern, ${ }^{2}$ Anthony Rodgers, ${ }^{2}$ \\ Aravinda Thiagalingam, ${ }^{3,4}$ Nicholas Graves, ${ }^{1}$ Clara K Chow ${ }^{2,3,4}$
}

- Additional material is published online only. To view please visit the journal online (http://dx.doi.org/10.1136/ heartjnl-2016-310195)

${ }^{1}$ Queensland University of Technology, Brisbane, Queensland, Australia

${ }^{2}$ The George Institute for Global Health, Sydney Medical School University of Sydney, Sydney, Australia

${ }^{3}$ Department of Cardiology, Westmead Hospital, Sydney, Australia

${ }^{4}$ Westmead Clinical School, University of Sydney, Sydney, Australia

Correspondence to

Clara K Chow, Cardiology, The George Institute for Global Health, PO Box M201, Missenden Road, Camperdown, Sydney, NSW 2050, Australia; cchow@georgeinstitute.org.au

Received 20 June 2016 Revised 29 November 2016 Accepted 27 December 2016 Published Online First 8 February 2017

\section{ABSTRACT \\ Objective To estimate the cost-effectiveness of Tobacco, Exercise and Diet Messages (TEXT ME), a text message-based intervention that provides advice, motivation, information and support to improve health- related behaviours.}

Methods A lifetime Markov model was used to estimate major vascular events (myocardial infarctions and strokes) avoided, quality-adjusted life years (QALYs) gained, costs to the health system and the incremental cost per QALY gained. The model was informed by data from a randomised controlled trial of TEXT ME, with evidence from systematic reviews and meta-analyses used to estimate the effects of changes in risk factors on the risk of major vascular events. Expected costs and health outcomes were estimated with uncertainty surrounding these characterised using probabilistic sensitivity analysis and a number of scenario analyses. Results For a target population of 50000 patients with documented coronary heart disease, the intervention is expected to lead to 563 fewer myocardial infarctions, 361 fewer strokes and 1143 additional QALYs. TEXT ME is expected to lead to an overall saving of $\$ 10.56$ million for the health system over the patients' lifetimes. The intervention can therefore be considered cost-saving and health-improving. Neither parameter nor structural uncertainty had a significant impact on the conclusion that TEXT ME is cost-effective.

Conclusions The provision of TEXT ME is predicted to lead to better health outcomes and an overall saving in costs for the health system.

Trial registration number anzctr.org.au identifier: ACTRN12611000161921.

\section{INTRODUCTION}

Cardiovascular disease (CVD), including coronary heart disease (CHD) and stroke, are the leading causes of lost years of healthy life globally. ${ }^{1}$ Smoking, physical inactivity and obesity increase the risk of developing CVD and of recurrent cardiovascular events among patients with a history of CVD. ${ }^{1}$ While adhering to behavioural recommendations improves health outcomes, ${ }^{2}$ the prevalence of healthy behaviours among those with a history of CHD is low, with poor knowledge of risk factors reported. ${ }^{3}$

Telehealth interventions are an effective means of delivering behavioural change disease populations. ${ }^{5}$ This prompted the development of the Tobacco, Exercise and Diet Messages (TEXT ME) intervention for patients with CHD, with the messages developed based on behaviour change theory, national guidelines and input from health professionals. ${ }^{6}$ TEXT ME has recently been assessed in a randomised controlled trial, where individuals with documented CHD were recruited through inpatient and outpatient cardiology services at a large tertiary referral centre in Sydney, Australia. $^{78}$

CVD prevention requires scalable approaches that provide clinical benefit and value, with benefits justifying the costs incurred. The aim of this study is to estimate the potential scaled-up and long-term benefits of the TEXT ME programme, and evaluate the value for money of the programme if it were provided in Australia.

\section{METHODS}

\section{Target population}

Based on the TEXT ME study eligibility criteria, ${ }^{8}$ the target population for the intervention is individuals with documented $\mathrm{CHD}$, defined as prior myocardial infarction (MI), coronary artery bypass graft surgery, percutaneous coronary intervention or $50 \%$ or greater stenosis in at least one major epicardial vessel on coronary angiography, who are admitted to Australian public hospitals. For this analysis, we considered the cost-effectiveness of the intervention if provided to a target population of 50000 patients. Given that over a million patients are admitted to hospital in Australia annually with CVD as either the principal or additional diagnosis, 50000 was considered to be a feasible target for the initial roll-out of the intervention across a state in Australia. $^{9}$

\section{Comparators}

TEXT ME consists of four text messages via short message service (SMS) per week for 24 weeks. The messages target multiple risk factors and provide advice, motivation, information and support to improve health behaviours (box 1). A bank of messages was developed with input from investigators, clinicians, academics and patients through a multistage iterative process, and for each participant, messages are selected depending on their key baseline characteristics, such as their smoking status. secondary prevention programmes to patients with $\mathrm{CVD},{ }^{4}$ and text message interventions have achieved behaviour change among other chronic The development of this intervention has previously been described in detail. ${ }^{7}$ As TEXT ME is supplementary to usual care, the costs and health outcomes

To cite: Burn E, Nghiem S, Jan ${ }^{2}$, et al. Heart 


\section{General cardiovascular health and medications}

- Check out http://www.heartfoundation.org.au for tips and info about preventing heart disease.

- Are you taking daily aspirin? If not, discuss it with your doctor.

- Not having support of family and friends can worsen heart disease - if you need help, don't be afraid to ask.

- Remember-cholesterol- and blood pressure-lowering tablets need to be taken every day.

\section{Nutrition}

- Healthy eating means at least five servings of vegetables and two servings of fruit every day.

- Try bananas for an easy and nutritious sandwich filling.

- To add interest to your meals, try a new fruit, vegetable or herb.

- Try steaming, baking or BBQ to reduce the need for excess oil when cooking.

\section{Physical activity}

- Keep a calendar of how often you walk.

- Begin activity at low intensity and gradually increase.

- There are many ways to increase your activity levels. Try Tai Chi, pilates, gardening, yoga or dancing.

- The more you eat, the more you need to exercise.

\section{Smoking}

- It's never too late to quit smoking.

- It may take several attempts to quit, so keep trying.

- If you crave a cigarette, try and distract yourself by going for a walk or doing something creative.

- Check out the website http://www.icanquit.com.au for tips and to track your progress when quitting smoking.

$\mathrm{BBQ}$, barbeque.

associated with providing TEXT ME alongside usual care were compared against those from providing usual care alone.

\section{Decision-analytical model}

A lifetime Markov model is used to provide the framework for the analysis. The model, shown in figure 1, consisted of six health states: history of CHD, MI, stroke, history of MI, history of stroke and dead. The model had a cycle length of 6 months. In line with a model developed to assess the impact of primary prevention, ${ }^{10}$ the key simplifying assumption was that individuals can only have one of either an MI or a stroke, after which they move to and then remain in the history of secondary event state until death.

\section{Health outcomes}

Quality-adjusted life years (QALYs) were the primary measure of health benefit. By estimating health effects in terms of QALYs, which provide a measure of both quantity and quality of life, the value for money of providing TEXT ME can be compared against that of providing a wide range of interventions across the health system. Life years and number of MIs and strokes for the cohort were also estimated under usual care and TEXT ME.

\section{Measurement of effectiveness}

The effectiveness of TEXT ME on risk factor levels was based on the findings of the randomised controlled trial, where 710 participants were enrolled and randomised into receiving the intervention or usual care, and data were collected at baseline and after 6 months. ${ }^{8}$

Relative risk reductions for MI and stroke were estimated on the basis of differences in risk factors observed within the trial (figure 2). Systematic reviews and meta-analyses were used, with these having estimated the relative risk reductions for MI and stroke associated with changes in low-density lipoprotein cholesterol (LDL-C), systolic blood pressure (SBP) and smoking (table 1). ${ }^{11-14}$ Relative risks were based on the size of the risk factor difference, with a log-linear relationship applied; for example, if an $\mathrm{X} \mathrm{mm} \mathrm{Hg}$ SBP reduction was estimated to lead to a relative risk of $Y$, then the relative risk for an SBP reduction of $\mathrm{Z}$ was estimated as $\mathrm{Y}^{\mathrm{Z} / \mathrm{X}}$. As no interaction or effect modification across these risk factors is known, the risk reductions associated with each modality were multiplied in order to estimate an overall relative risk reduction.

Relative risk reductions associated with the intervention were reduced by $25 \%$ over the first year to take into account an expected lag in intervention effects. Given that the risk factor differences were only observed at 6 months, these were required to be extrapolated into the future. To take into account the likely drop off of intervention effects, in the base case analysis, it was assumed that any relative risk reductions estimated at 6 months would steadily diminish until being entirely mitigated after 5 years.

The risks for major vascular events under usual care were based on the number of events observed in the control arms of

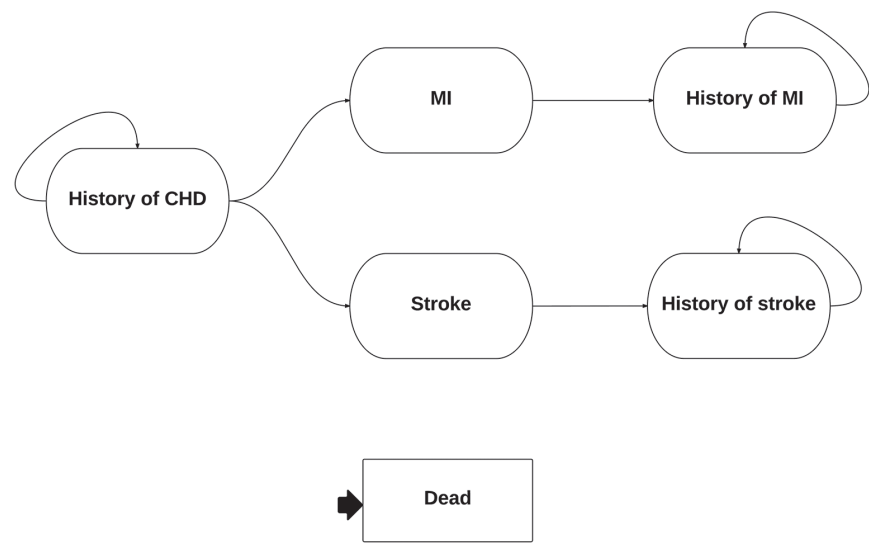

Figure 1 State-based Markov model. Individuals begin in the history of coronary heart disease (CHD) state and either remain there until death or move to either of the myocardial infarction (MI) or stroke states, after which they either die or move to the history of MI or stroke state, in which they would stay until death. 


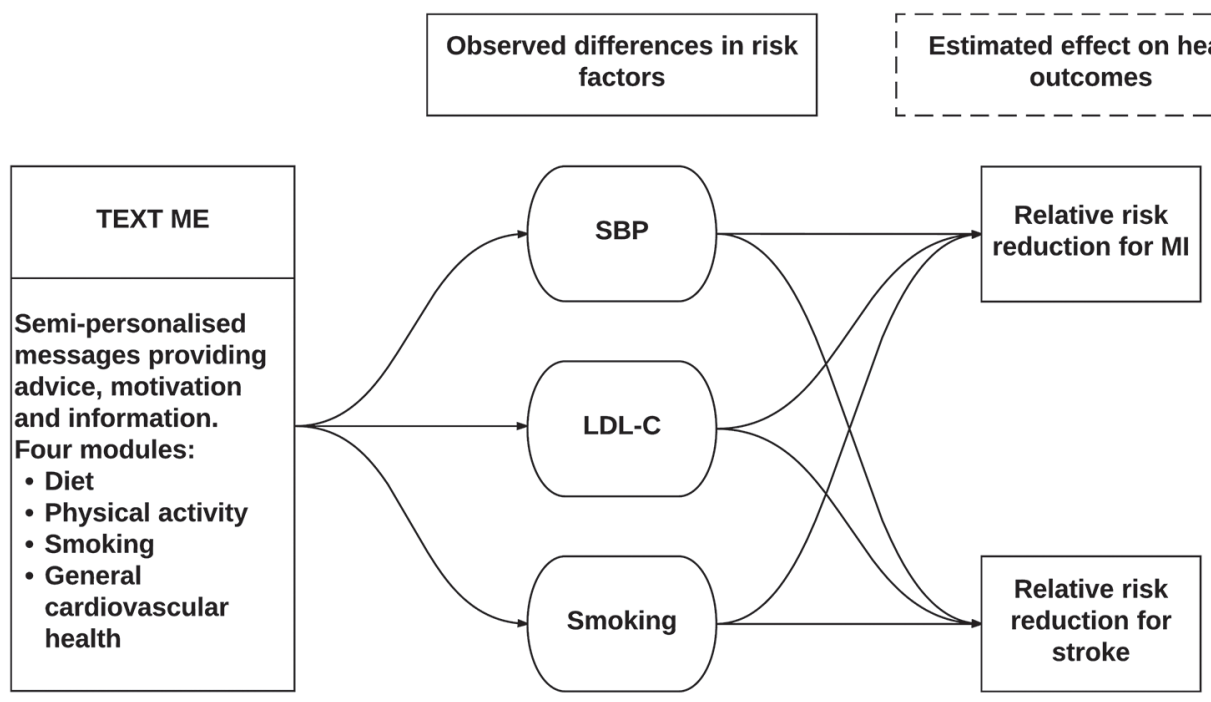

Figure 2 Estimated effect of TEXT ME on health outcomes. Differences in risk factors were observed at 6 months (table 2). Relative risk reductions associated with changes in these risk factors (table 1) were estimated and extrapolated into the future. LDL-C, low-density lipoprotein cholesterol; MI, myocardial infarction; SBP, systolic blood pressure; TEXT ME, Tobacco, Exercise and Diet Messages.

randomised trials of LDL-C-lowering statin therapies for individuals at high risk, with $4.8 \%$ and $4.9 \%$ risks of MI events observed annually for men and women, respectively, and $1.6 \%$ and $2.2 \%$ for strokes. ${ }^{11}$ The risk of death following major vascular events was based on age-specific survival rates reported for Australia. ${ }^{15}$ In the absence of an event, the risk of death was based on Australian life tables, ${ }^{16}$ which were doubled, in line with economic evaluations with similar patient cohorts, ${ }^{17}$ to reflect the greater mortality rates for patients with a history of CHD. A risk of death of 1 was assumed if individuals reached 100 years old.

\section{Measurement and valuation of preference-based outcomes}

The SF-12 survey was undertaken at baseline and 6 months in the TEXT ME trial, with data collectors blinded to treatment assignment. $^{8}$ These responses were transformed into SF-6D using the algorithm provided by Brazier et al, ${ }^{18}$ and, as SF-6D at 6 months was similar between treatment groups, these scores were pooled and used to inform the estimated quality of life of the history of CHD state.

Quality of life associated with a MI event was based on scores reported 3 months following a MI observed within an RCT setting in the UK. ${ }^{19}$ In line with previous economic evaluations, ${ }^{20}$ it was assumed that quality of life score for the history of MI would be equal to that of the history of CHD state subsequently. Meanwhile, quality of life associated with stroke was informed by scores reported 3 months following an ischaemic stroke within an observational cohort of patients, while the history of stroke state was based on the 6-month scores from the same patients. ${ }^{21}$

\section{Estimating resources and costs}

A health system perspective was taken, with only those costs being borne by the health system included in the analysis. The costs associated with the provision of the TEXT ME programme were estimated in consultation with the programme staff. They used their administrative records and experience from undertaking the trial to estimate the volume of resources required and market prices. As well as the cost of providing the intervention, costs due to healthcare utilisation were also incorporated. Primary care costs were based on records from the Medicare Benefits Schedule and the Pharmaceutical Benefits Scheme, which contain records of primary care consultations and prescriptions, respectively, of the trial participants in 2014. Meanwhile, hospital costs relating to major vascular events were based on the age- and gender-specific cost per hospital separation reported for Australia. ${ }^{22}$ All costs are reported

Table 1 Relative risks associated with changes in systolic blood pressure and LDL-C

\begin{tabular}{|c|c|c|c|c|c|}
\hline Risk factor & Risk factor change & $\begin{array}{l}\text { RR for patients with prior } \\
\text { myocardial Infarction }\end{array}$ & $\begin{array}{l}\text { Probability distribution } \\
\text { around the mean }\end{array}$ & RR for stroke & $\begin{array}{l}\text { Probability distribution } \\
\text { around the mean }\end{array}$ \\
\hline$S B P * 12$ & $10 \mathrm{~mm} \mathrm{Hg}$ reduction & $0.76(0.68$ to 0.86$)$ & Normal $(0.76,0.05)$ & $0.65(0.53$ to 0.80$)$ & Normal $(0.65,0.07)$ \\
\hline \multicolumn{6}{|l|}{$\mathrm{LDL}-\mathrm{C}^{11}$} \\
\hline Men & $1 \mathrm{mmol} / \mathrm{L}$ reduction & $0.74(0.70$ to 0.78$)$ & Normal $(0.74,0.02)$ & $0.83(0.76$ to 0.90$)$ & Normal $(0.83,0.04)$ \\
\hline Women & $1 \mathrm{mmol} / \mathrm{L}$ reduction & 0.83 (0.74 to 0.93$)$ & Normal $(0.83,0.05)$ & 0.90 (0.78 to 1.04$)$ & Normal $(0.83,0.07)$ \\
\hline \multicolumn{6}{|l|}{ Smoking ${ }^{1314}$} \\
\hline Men & Quitting & $0.68(0.57$ to 0.82$)$ & Normal $(0.68,0.06)$ & $0.60(0.53$ to 0.67$)$ & Normal $(0.60,0.04)$ \\
\hline Women & Quitting & $0.68(0.57$ to 0.82$)$ & Normal $(0.68,0.06)$ & 0.55 (0.47 to 0.63$)$ & Normal $(0.55,0.04)$ \\
\hline
\end{tabular}

*Independent of class of blood pressure-lowering drug.

tIndependent of type of statin.

For RRs, parentheses contain the $95 \% \mathrm{Cl}$ used for sensitivity analyses. For probability distributions around expected (mean) effects, the first and second values in parentheses correspond to mean and SE in normal distribution.

LDL-C, low-density lipoprotein cholesterol; RR, relative risk; SBP, systolic blood pressure. 


\begin{tabular}{|c|c|c|}
\hline & $\begin{array}{l}\text { Expected (mean) } \\
\text { value }\end{array}$ & $\begin{array}{l}\text { Probability distribution } \\
\text { around the mean }\end{array}$ \\
\hline Cohort size & 50000 & - \\
\hline \multicolumn{3}{|l|}{ Patient characteristics } \\
\hline Gender: male & $82 \%$ & $\beta(516,116)$ \\
\hline \multicolumn{3}{|l|}{ Age } \\
\hline Male & 58 & Normal $(58,0.40)$ \\
\hline Female & 57 & Normal $(57,0.88)$ \\
\hline \multicolumn{3}{|l|}{ Quality of life } \\
\hline History of CHD & 0.74 & $\beta(18479,6469)$ \\
\hline MI & 0.67 & $\beta(144,71)$ \\
\hline History of Ml & 0.74 & $\beta(18479,6469)$ \\
\hline Stroke & 0.62 & $\beta(159,101)$ \\
\hline History of stroke & 0.68 & $\beta(115,71)$ \\
\hline \multicolumn{3}{|l|}{ Costs } \\
\hline Primary care costs* & 2,095 & $\gamma(147,14)$ \\
\hline Cost per $\mathrm{MI}^{*}$ & 11877 & $\gamma(400,30)$ \\
\hline Cost per stroke* & 18801 & $\gamma(400,47)$ \\
\hline \multicolumn{3}{|l|}{ Intervention effects } \\
\hline \multicolumn{3}{|l|}{ Change in SBP $(\mathrm{mm} \mathrm{Hg}) \dagger$} \\
\hline Male & -7.7 & Normal $(-7.7,1.3)$ \\
\hline Female & -8.2 & Normal $(-8.1,2.5)$ \\
\hline \multicolumn{3}{|l|}{ Change in LDL-C (mmol/L)† } \\
\hline Male & -0.1 & Normal $(-0.1,0.1)$ \\
\hline Female & -0.1 & Normal $(-0.1,0.1)$ \\
\hline \multicolumn{3}{|c|}{ Change in proportion of cohort smoking $\neq$} \\
\hline Male & $-15 \%$ & Normal $(-15 \%, 0.04)$ \\
\hline Female & $-26 \%$ & Normal $(-26 \%, 0.07)$ \\
\hline \multicolumn{3}{|l|}{ Intervention cost } \\
\hline Database/IT infrastructure & 75000 & \\
\hline Project manager & 130000 & \\
\hline Clinical advisor & 26000 & \\
\hline Patient enrolment & 846354 & \\
\hline Messages & 480000 & \\
\hline $\begin{array}{l}\text { Additional (miscellaneous } \\
\text { expenses) }\end{array}$ & 100000 & \\
\hline Total cost & 1657354 & U (1 243 016, 2071 693) \\
\hline \multicolumn{3}{|c|}{$\begin{array}{l}\text { *For 55-64 male, for full primary care and hospital costs, see Appendix. } \\
\text { tAnalysis of covariance was used and included randomised groups (usual care and } \\
\text { TEXT ME) and baseline values. } \\
\text { łTest of proportions. For probability distributions around the mean, the first and } \\
\text { second values in parentheses correspond to mean and SE in normal distribution and } \\
\text { alpha and beta in beta ( }(\beta), \text { gamma }(\gamma) \text { and uniform (U) distributions. } \\
\text { CHD, coronary heart disease; LDL-C, low-density lipoprotein cholesterol; MI, } \\
\text { myocardial infarction; SBP, systolic blood pressure; TEXT ME, Tobacco, Exercise and } \\
\text { Diet Messages. }\end{array}$} \\
\hline
\end{tabular}

in 2014 Australian dollars. In line with guidelines for Australia, both future costs and QALYs were discounted by $3 \%$ annually.

\section{Analytical methods}

The decision-analytical model was used to estimate expected (mean) costs and health outcomes, with the uncertainty around these expected effects estimated. Expected results were given with all parameters held at their mean values and under base case assumptions. Uncertainty around the expected (mean) effects was assessed using probabilistic sensitivity analysis, with input parameters assigned probability distributions and 1000 Monte Carlo simulations conducted based on these distributions and SEs. The sets of estimated costs and QALYs from each simulations are shown, alongside the expected results, on a cost-effectiveness plane. This approach captures parameter uncertainty, uncertainty in the estimation of the parameter of interest, but does not assess stochastic uncertainty, random variability in outcomes, or patient heterogeneity, the variability between individuals, which can be attributed to given characteristics. ${ }^{23}$

The timing over which an investment in TEXT ME would become cost-effective was considered using a net benefit curve. ${ }^{24}$ This plots the expected net health benefit, which is equal to the expected change in health outcomes minus the change in costs divided by the cost-effectiveness threshold, associated with providing the intervention over the time horizon of the analysis. The cost-effectiveness threshold used was $\$ 64000$, which has been estimated to be the willingness to pay for an additional QALY in Australia. ${ }^{25}$ When net health benefit is positive, it indicates that the intervention is cost-effective over the given time horizon; for example, if net benefit is positive at 5 years, this implies that the health benefits that accrue over these 5 years merit the costs incurred up to this point.

Several tests were conducted on the sensitivity of results to changes in structural assumptions with the analysis re-run under alternative scenarios conservative against TEXT ME. First, relative risk reductions were assumed to be mitigated entirely after 1 year rather than 5 years. Second, the baseline risk of major vascular events was reduced by $50 \%$. Third, the estimated differences in risk factors were held at their low values from 95\% CI. Fourth, the expected cost of TEXT ME is doubled. These scenarios were then run together. In addition, a one-way sensitivity analysis was undertaken to see by how much the intervention cost would need to change for the result of the evaluation to alter.

\section{RESULTS}

\section{Study parameters}

The characteristics of the study population are detailed in table 2 . The provision of TEXT ME was expected to lead to a reduction in LDL-C, SBP and smoking. For example, for women, TEXT ME was associated with an expected reduction of 8.16 in SBP, $0.1 \mathrm{mmol} / \mathrm{L}$ for LDL-C and a $26 \%$ reduction in the proportion of smokers (table 2). This would be expected to lead to an expected relative risk of 0.72 for $\mathrm{MI}$ and 0.61 for a stroke (table 1 ), all else equal.

Delivering the intervention involves setting up IT servers and cloud space to manage the data at an expected annual cost of Australian $\$ 75000$. A project manager working full time and a clinical advisor spending a fifth of their time on the intervention would be required, and these were expected to be receiving salaries of $\mathrm{A} \$ 100000$ and $\mathrm{A} \$ 50000$, respectively, both with 30\% on-costs. Clinical staff would be required to enrol patients, and this was expected to take $15 \mathrm{~min}$ per participant. Assuming clinical staff received a salary of $\mathrm{A} \$ 100000$ with $30 \%$ on-costs and 4 weeks of holiday, each 15 min would cost $\mathrm{A} \$ 17$ and enrolment of the entire cohort would cost $\$ 846354$. The expected cost of sending an SMS was $\$ 0.10$, and with participants receiving 96 messages over the course of the intervention, the cost of sending SMS messages to the cohort was expected to be $\mathrm{A} \$ 480000$. A further $\mathrm{A} \$ 100000$ was expected to be budgeted for any miscellaneous expenses. The intervention as a whole was therefore expected to have a total cost of $\$ 1.66$ million or $\mathrm{A} \$ 33$ per participant (table 2).

\section{Incremental costs and outcomes}

Under base case assumptions, men had a mean life expectancy of 14.21 if receiving usual care and 14.23 if receiving TEXT ME, while women had a life expectancy of 17.45 and 17.48 , respectively. The provision of TEXT ME was estimated to lead to 563 fewer MIs and 361 fewer strokes over the course of these years. 


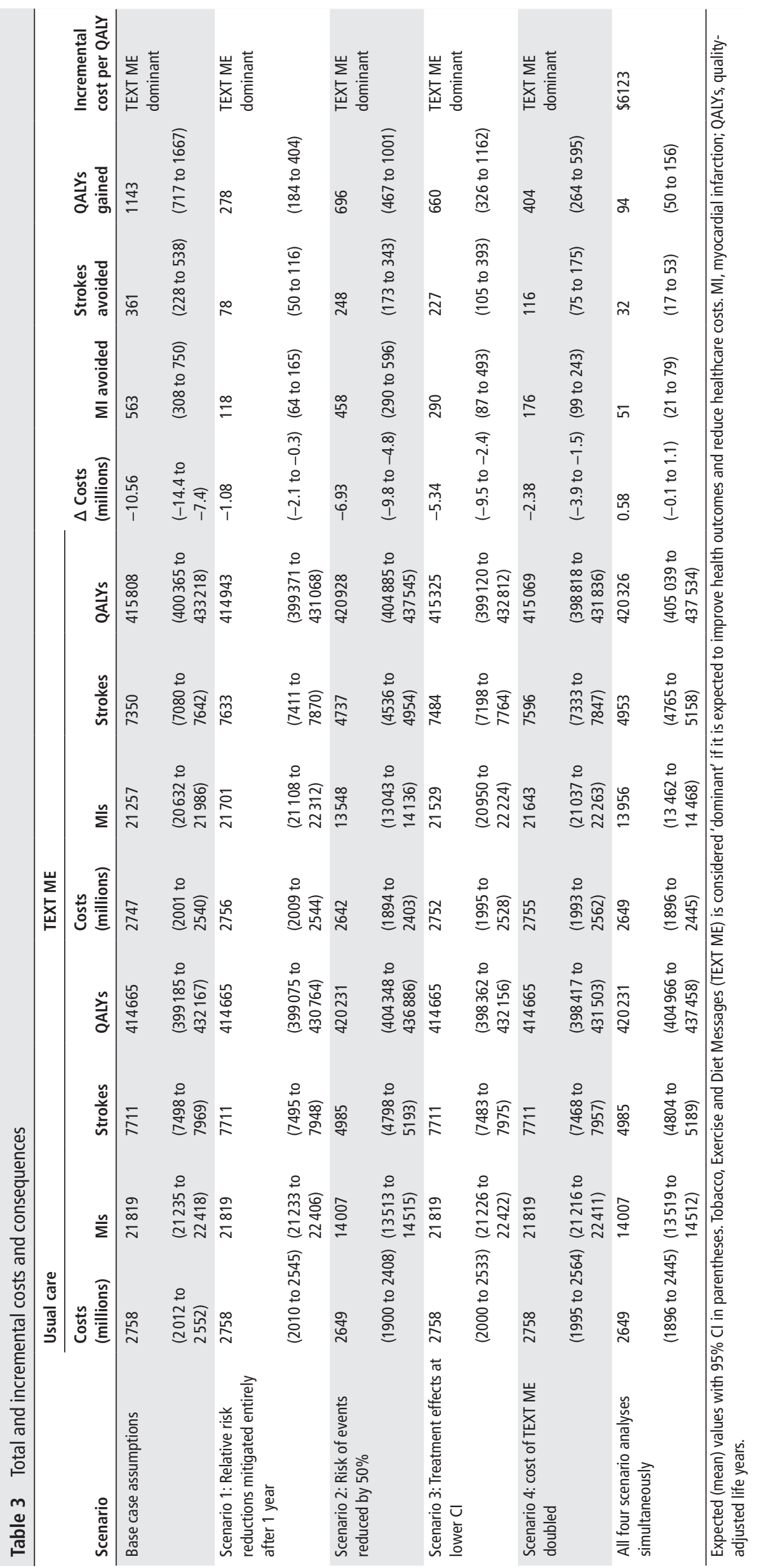




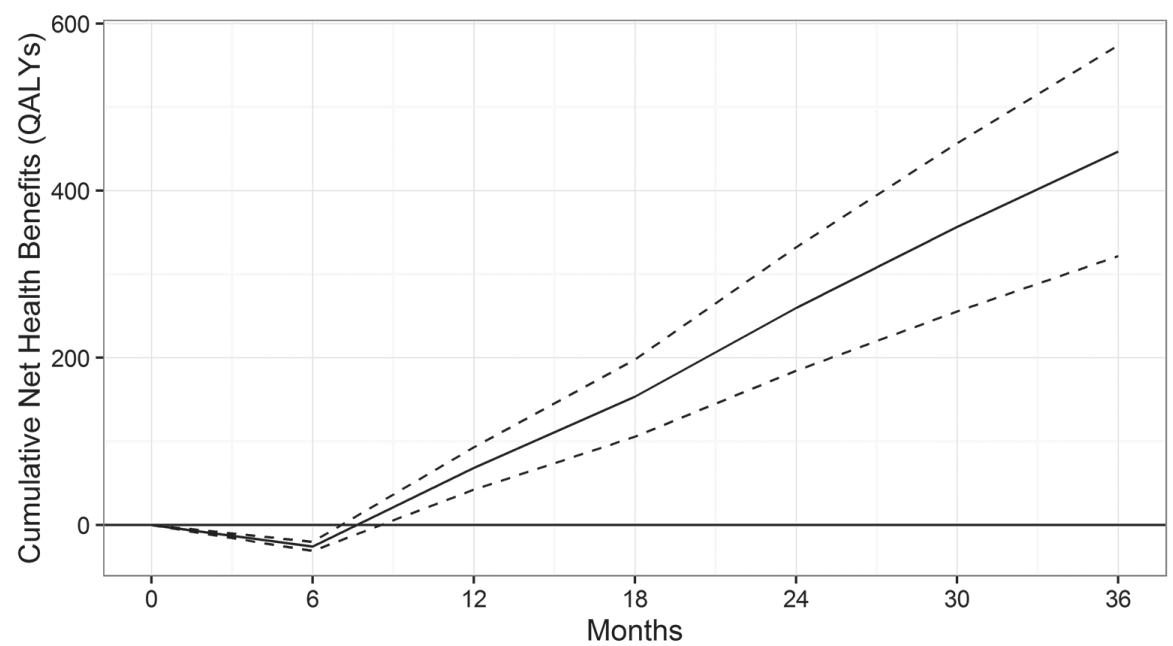

Figure 3 Net benefit curve. The timing over which an investment in TEXT ME would become cost-effective given a cost-effectiveness threshold used is $\$ 64000$. QALYs, quality-adjusted life years; TEXT ME, Tobacco, Exercise and Diet Messages.

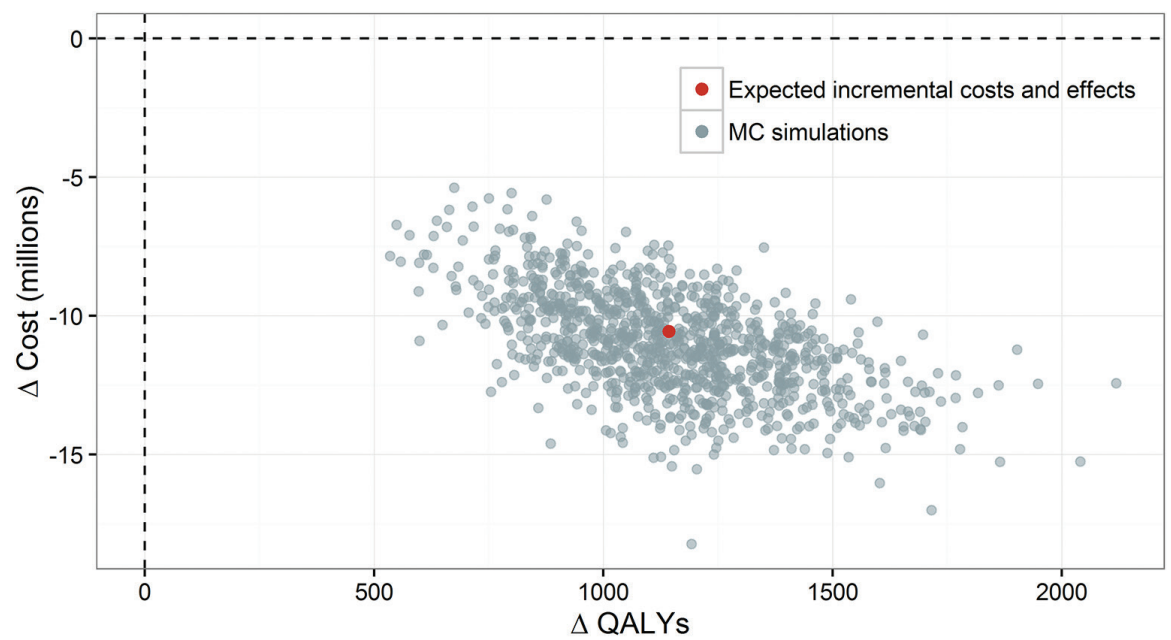

Figure 4 Cost-effectiveness plane. The sets of estimated costs and quality-adjusted life years (QALYs) from each of the $1000 \mathrm{Monte}$ Carlo (MC) simulations are shown with the expected results.

As a result, the intervention was expected to lead to 1006 additional life years and a gain of 1143 QALYs (1469 if undiscounted). While the provision of TEXT ME was expected to cost Australian $\$ 1.66$ million (table 2), the reduction in major vascular events (MIs and strokes) was expected to lead to an overall cost-saving of A \$10.56 million (table 3). TEXT ME can therefore be considered 'dominant', with it expected to lead to better health outcomes and cost-savings. Indeed, the intervention can be considered costeffective even after accounting for only the improvements in health outcomes which occur over the first year following its provision (figure 3).

\section{Parameter uncertainty}

Parameter uncertainty had little effect on the conclusion that TEXT ME is cost-effective. Under base case assumptions, the intervention was health-improving and cost-saving given each of the sets of simulated costs and effects (figure 4).

\section{Structural uncertainty}

Under all of the individual scenario analyses, TEXT ME was expected to be cost-saving and health-improving (table 3). When all scenarios were run simultaneously TEXT ME was no longer expected to be cost-saving but would still be considered cost-effective given a willingness to pay for an additional QALY of $\$ 64000 .^{25}$ Under base case assumptions, TEXT ME was costsaving if the intervention cost was less than $\$ 245$ per participant and cost-effective given a cost of less than A $\$ 1750$ per participant.

\section{DISCUSSION}

In Australia, the provision of TEXT ME was expected to lead to be health-improving and cost-saving, with the benefits of the intervention quickly outweighing its costs. Sensitivity analyses found that sources of uncertainty had little impact on the conclusion that TEXT ME was a cost-effective use of healthcare resources for the cohort as a whole.

Economic evaluations of CVD management programmes have varied in methodologies, making direct comparisons difficult, but a number of other evaluations have also found secondary prevention programmes to be cost-effective. ${ }^{26}$ While two other telehealth interventions, both of which are based on one-to-one telephone calls, have been estimated to be health-improving, ${ }^{26}$ they have been found to be costly to provide. Text message-based intervention, such as TEXT ME, therefore, seems to offer a more cost-effective method of delivering a telehealth intervention for such patients. Indeed, participants in the trial of TEXT ME were engaged with and positive about the programme, with 
this due to the programme being initiated close to the time of a cardiovascular event, receiving consistent messages following in-hospital education and management, having the opportunity for face-to-face contact at programme commencement and the messages being personalised. ${ }^{27}$

The principal limitations of this evaluation are due to the requirement to extrapolate changes in risk factors observed after 6 months into the future and to link these changes to risk reductions for major vascular events. The model used allows such an extrapolation but is a simplification of reality allowing individuals to have only one future major vascular event. There is no way of knowing with certainty how differences will be maintained into the future, and the base case assumption that any relative risk reductions would steadily diminish until being eliminated after 5 years is necessarily a subjective estimate. In addition, the impact of events on quality of life is based on the literature, while background mortality is expected to be in line with general population life tables, which have been doubled to reflect the greater morbidity of the patient cohort. Given that these estimates are based on distinct patient populations, they may not truly reflect what would be realised by the model cohort. While significant parameter uncertainty undoubtedly surrounds such inputs, sensitivity analyses have been undertaken, which show the conclusions of this evaluation to be robust. Structural uncertainty has a more serious impact on the conclusions; however, with the intervention no longer expected to be cost-saving if risk reductions are mitigated entirely after 1 year, the baseline risk of events is halved, and realised treatment effects are at the lower CI of those estimated in the trial and the cost of the intervention is doubled. Costs and outcomes are estimated for the cohort as a whole, and it should be noted that individual results would vary due to both random variability and variability between individuals, which can be attributed to given characteristics.

The benefits of the intervention may in fact be underestimated given that only two types of vascular events, MI and stroke, were included in the model. Any sustained changes in risk factors would reduce the risk for any number of future events; however, for model parsimony, all possible future events were not included. In addition, the effect of differences in body mass index and physical activity, which were observed in the trial of TEXT ME, ${ }^{8}$ both of which have been shown to have an impact on health outcomes related to CVD, ${ }^{28} 29$ were not incorporated in the analysis. As well as reducing the risk of vascular events, changes in these risk factors would likely lead to reduced healthcare costs, for example, with fewer antihypertensives prescribed. In addition, MIs were only expected to reduce quality of life over the subsequent 6 months, which is likely to underestimate the impact of such events.

TEXT ME is predicted here to lead to better health outcomes and reduced healthcare costs in Australia. Given the low prevalence of healthy behaviours among those with a history of CHD across a wide range of countries ${ }^{30}$ and the substantial proportion of the global burden of disease caused by CVD, ${ }^{1}$ the results here are likely to be of relevance for all health systems. Telemedicine and, in particular, SMS-based interventions provide an affordable and scalable means for delivering health interventions. This research suggests they can also provide good value for money.

Acknowledgements The authors thank Laura De Keizer, research assistant on the TEXT ME study, who coordinated applications to obtain linked health record data for use in the current analyses.

Contributors EB, SN supervised by NG conducted the economic analysis. EB and CC drafted the paper. JR, SF, AR, AT and CC (principal investigator) were involved

\section{Key messages}

\section{What is already known about this subject?}

mHealth interventions have shown promise in being able to improve cardiovascular risk factor levels in patients with cardiovascular disease or at high cardiovascular risk. While they appear to be potentially low-cost interventions to deliver, formal costeffectiveness evaluations are required to support the uptake of new interventions by healthcare payers.

\section{What does this study add?}

This study provides information on the cost-effectiveness of a secondary prevention programme (TEXT ME) delivered by mobile phone text message to patients with coronary heart disease. Delivery of the TEXT ME intervention is predicted to lead to better health outcomes and an overall saving for the health system, given the major vascular events avoided and the relatively low costs of the intervention.

\section{How might this impact on clinical practice?}

These findings can prompt healthcare providers to implement text message-based prevention programmes, which should lead to patients gaining access to those outside of research programmes.

in the design, implementation and interpretation of the TEXT ME trial. All authors contributed to reviewing and commenting on this paper and analyses.

Funding This health economic evaluation was made possible through funding support from a peer-reviewed competitive award from the BUPA Foundation. The TEXT ME study was supported by the National Heart Foundation of Australia Grantin-Aid (G10S5110). Dr ChowCKC is funded by a Career Development Fellowship cofunded by the National Health and Medical Research Council (NHMRC) (1033478) and National Heart Foundation (11 S6016) and Sydney Medical Foundation Chapman Fellowship. Dr RedfernJR is funded by an NHMRC Career Development Fellowship (1061793) cofunded with a National Heart Foundation Future Fellowship (G160523). None of the funders had any role in the design, analysis or writing or approving of the manuscript prior to submission. This work was supported by peer-reviewed grants from the National Heart Foundation of Australia Grant-in-Aid (G10S5110) and a BUPA Foundation Grant.

Competing interests None declared.

Provenance and peer review Not commissioned; externally peer reviewed.

(C) Article author(s) (or their employer(s) unless otherwise stated in the text of the article) 2017. All rights reserved. No commercial use is permitted unless otherwise expressly granted.

\section{REFERENCES}

1 Murray CJ, Vos T, Lozano R, et al. Disability-adjusted life years (DALYs) for 291 diseases and injuries in 21 regions, 1990-2010: a systematic analysis for the Global Burden of Disease Study 2010. Lancet 2012;380:2197-223.

2 Chow CK, Jolly S, Rao-Melacini P, et al. Association of diet, exercise, and smoking modification with risk of early cardiovascular events after acute coronary syndromes. Circulation 2010;121:750-8

3 Redfern J, Ellis ER, Briffa T, et al. High risk-factor level and low risk-factor knowledge in patients not accessing cardiac rehabilitation after acute coronary syndrome. Med $\mathrm{J}$ Aust 2007;186:21-5.

4 Neubeck L, Redfern J, Fernandez R, et al. Telehealth interventions for the secondary prevention of coronary heart disease: a systematic review. Eur J Cardiovasc Prev Rehabil 2009;16:281-9

5 Fjeldsoe BS, Marshall AL, Miller YD. Behavior change interventions delivered by mobile telephone short-message service. Am J Prev Med 2009;36:165-73.

6 Redfern J, Thiagalingam A, Jan S, et al. Development of a set of mobile phone text messages designed for prevention of recurrent cardiovascular events. Eur J Prev Cardiol 2014;21:492-9.

7 Chow CK, Redfern J, Thiagalingam A, et al. Design and rationale of the tobacco, exercise and diet messages (TEXT ME) trial of a text message-based intervention for ongoing prevention of cardiovascular disease in people with coronary disease: a randomised controlled trial protocol. BMJ Open 2012;2:e000606.

8 Chow CK, Redfern J, Hillis GS, et al. Effect of lifestyle-focused text messaging on risk factor modification in patients with coronary heart disease: a randomized clinical trial. JAMA 2015;314:1255. 
9 AlHW. Diabetes and chronic kidney disease: Australian facts: morbidity-hospital care. Cardiovascular, Diabetes and Chronic Kidney Disease Series. Canberra: AlHW, 2014.

10 Lewsey JD, Lawson KD, Ford I, et al. A cardiovascular disease policy model that predicts life expectancy taking into account socioeconomic deprivation. Heart 2015;101:201-8.

11 Cholesterol Treatment Trialists.Efficacy and safety of LDL-lowering therapy among men and women: meta-analysis of individual data from 174000 participants in 27 randomised trials. The Lancet 2015;385:1397-405.

12 Law MR, Morris JK, Wald NJ. Use of blood pressure lowering drugs in the prevention of cardiovascular disease: meta-analysis of 147 randomised trials in the context of expectations from prospective epidemiological studies. BMJ 2009;338:b1665.

13 Critchley JA, Capewell S. Mortality risk reduction associated with smoking cessation in patients with coronary heart disease: a systematic review. JAMA 2003;290:86-97.

14 Peters SA, Huxley RR, Woodward M. Smoking as a risk factor for stroke in women compared with men: a systematic review and meta-analysis of 81 cohorts, including 3,980,359 individuals and 42,401 strokes. Stroke 2013;44:2821-8.

15 Department of Health and Welfare. Age-standardised proportion of people aged 40-90 years surviving a major coronary event by sex 1994-2009. 2014.

16 Australian Bureau of Statistics. Life Tables, States, Territories and Australia, 2011-2013, 2014:3302055001.

17 National Institute for Health and Care Excellence. Myocardial infarction: cardiac rehabilitation and prevention of further cardiovascular disease. Clinical Guideline [CG172]. London: NICE, 2013.

18 Brazier JE, Roberts J. The estimation of a preference-based measure of health from the SF-12. Med Care 2004:42:851-9.

19 Lacey EA, Musgrave RJ, Freeman JV, et al. Psychological morbidity after myocardial infarction in an area of deprivation in the UK: evaluation of a self-help package. Eur J Cardiovasc Nurs 2004;3:219-24.

20 Becerra V, Gracia A, Desai K, et al. Cost-effectiveness and public health benefit of secondary cardiovascular disease prevention from improved adherence using a polypill in the UK. BMJ Open 2015;5:e07111.
21 Pickard AS, Johnson JA, Feeny DH, et al. Agreement between patient and proxy assessments of health-related quality of life after stroke using the EQ-5D and health utilities index. Stroke 2004;35:607-12.

22 AlHW. Health care expenditure on cardiovascular diseases. Cat No CVD 65. Canberra: AlHW ,2008-09.

23 Briggs AH, Weinstein MC, Fenwick EA, et al; ISPOR-SMDM Modeling Good Research Practices Task Force. Model parameter estimation and uncertainty analysis: a report of the ISPOR-SMDM Modeling good Research Practices Task Force working Group-6. Med Decis Making 2012;32:722-32.

24 McCabe C, Edlin R, Hall P. Navigating time and uncertainty in health technology appraisal: would a map help? Pharmacoeconomics 2013;31:731-7.

25 Shiroiwa T, Sung YK, Fukuda T, et al. International survey on willingness-to-pay (WTP) for one additional QALY gained: what is the threshold of cost effectiveness? Health Econ 2010;19:422-37.

26 Maru S, Byrnes J, Whitty JA, et al. Systematic review of model-based analyses reporting the cost-effectiveness and cost-utility of cardiovascular disease management programs. Eur J Cardiovasc Nurs 2015;14:26-33.

27 Redfern J, Santo K, Coorey G, et al. Factors influencing engagement, perceived usefulness and behavioral mechanisms associated with a text message support program. PLoS One 2016;11:e0163929.

28 Prospective Studies Collaboration. Body-mass index and cause-specific mortality in 900000 adults: collaborative analyses of 57 prospective studies. Lancet 2009;373:1083-96.

29 Sattelmair J, Pertman J, Ding EL, et al. Dose response between physical activity and risk of coronary heart disease: a meta-analysis. Circulation 2011;124:789-95.

30 Teo K, Lear S, Islam S, et al; PURE Investigators. Prevalence of a healthy lifestyle among individuals with cardiovascular disease in high-, middle- and low-income countries: the Prospective Urban Rural Epidemiology (PURE) study. JAMA 2013:309:1613-21. 